\title{
Epidemic Louse-Borne Typhus
}

National Cancer Institute

\section{Source}

National Cancer Institute. Epidemic Louse-Borne Typhus. NCI Thesaurus. Code C84689.

A gram-negative bacterial infection caused by Rickettsia prowazekii. It is spread by lice infected with the bacteria. Signs and symptoms include sudden headache, generalized muscle pain, malaise, and macular skin lesions. The infection may affect the central nervous system causing encephalitis. 